\title{
COUNTEREXAMPLES TO SOME POINTWISE ESTIMATES OF THE MAXIMAL CAUCHY TRANSFORM IN TERMS OF THE CAUCHY TRANSFORM
}

\author{
Daniel Girela-Sarrión \\ Universitat Autònoma de Barcelona, Departament de Matemàtiques \\ 08193 Bellaterra (Barcelona), Spain; dgirela@mat.uab.cat
}

\begin{abstract}
Motivated by the work of Mateu, Orobitg, Pérez and Verdera, who proved inequalities of the form $T_{*} f \lesssim M(T f)$ or $T_{*} f \lesssim M^{2}(T f)$ for certain singular integral operators $T$, such as the Hilbert or the Beurling transforms, we study the possibility of establishing this type of control when $T$ is the Cauchy transform along a Lipschitz graph. We show that this is not possible in general, and we give a partial positive result when the graph is substituted by a Jordan curve.
\end{abstract}

\section{Introduction}

Given a complex Radon measure $\nu$ on the plane $\mathbf{R}^{2} \equiv \mathbf{C}$, we define the Cauchy transform of $\nu$ by

$$
C \nu(z)=\int_{\mathbf{C}} \frac{d \nu(\xi)}{\xi-z}, \quad z \in \mathbf{C} \backslash \operatorname{supp}(\nu) .
$$

The integral in the definition may not be absolutely convergent if $z \in \operatorname{supp}(\nu)$. For this reason, we consider the truncated operators $\left\{C_{\epsilon}\right\}_{\epsilon>0}$, which are given by

$$
C_{\epsilon} \nu(z)=\int_{|\xi-z|>\epsilon} \frac{d \nu(\xi)}{\xi-z}, \quad z \in \mathbf{C} .
$$

Notice that the integral above is absolutely convergent for all $z \in \mathbf{C}$ if, for example, $|\nu|(\mathbf{C})<\infty$.

If $\mu$ is a fixed positive Radon measure in $\mathbf{C}$ and $f \in L_{\text {loc }}^{1}(\mu)$, we set

$$
C_{\mu} f(z)=C(f d \mu)(z), \quad z \in \mathbf{C} \backslash \operatorname{supp}(f d \mu)
$$

and, for $\epsilon>0$,

$$
C_{\mu, \epsilon} f(z)=C_{\epsilon}(f d \mu)(z), \quad z \in \mathbf{C} .
$$

We say that $C$ is bounded between two spaces of functions $X$ and $Y$ (typically, $L^{p}(\mu$ ), $\left.L^{p, \infty}(\mu), \ldots\right)$ if the operators $C_{\mu, \epsilon}$ are bounded from $X$ to $Y$ uniformly on $\epsilon>0$.

A particularly interesting case is the one that arises when $\mu$ is the arc-length measure (or some measure comparable to this) supported on a Lipschitz graph. To

doi:10.5186/aasfm.2013.3828

2010 Mathematics Subject Classification: Primary 42B20.

Key words: Calderón-Zygmund theory, Cauchy transform, Cotlar's inequality.

I wish to thank my advisor, $\mathrm{X}$. Tolsa, for his valuable guidance and support during the preparation of this work. I also want to express my gratitude to J. Mateu and J. Verdera for their interest on my work and for useful suggestions. Finally, I wish to thank the referees for their kind advice. The author was supported by Project MTM2010-16232 (Spain), Project 2009SGR-000420 (Generalitat de Catalunya) and funded by the European Research Council under the European Union's Seventh Framework Programme (FP7/2007-2013)/ERC Grant agreement no. 320501. 
be more precise, let $A: \mathbf{R} \rightarrow \mathbf{R}$ be a Lipschitz function, and let $\Gamma \subset \mathbf{R}^{2} \equiv \mathbf{C}$ be its graph, which we parametrize by

$$
z(x)=x+i A(x), \quad x \in \mathbf{R} .
$$

We define a measure $\mu$ on $\Gamma$ by

$$
\mu(z(E))=|E|,
$$

where $E$ is any Borel subset of $\mathbf{R}$. We will normally call $C_{\mu}$ the Cauchy transform along $\Gamma$. Recall that, since $A$ is Lipschitz, it is differentiable almost everywhere and, furthermore, its Lipschitz constant coincides with $\left\|A^{\prime}\right\|_{\infty}$. Moreover, it is easy to check that the measure $\mu$ that we are considering is comparable to the arc-length measure on $\Gamma$.

In [1], Calderón proved that $C_{\mu}$ is bounded in $L^{2}(\mu)$ when $\left\|A^{\prime}\right\|_{\infty}$ is sufficiently small. Later, in [2], Coifman, McIntosh and Meyer proved that $C_{\mu}$ is bounded in $L^{2}(\mu)$ for every Lipschitz function $A$. It also follows from the work of Calderón that

$$
\text { p.v. } C_{\mu} f(z)=\lim _{\epsilon \searrow 0} C_{\mu, \epsilon} f(z)
$$

exists for a.e. $z \in \operatorname{supp}(\mu)$ for all $f \in L^{2}(\mu)$, and, as a result, we can think of $C_{\mu}$ to be defined as a principal value operator

$$
C_{\mu} f(z)=\text { p.v. } \int_{\Gamma} \frac{f(\xi)}{\xi-z} d \mu(\xi) .
$$

All the considerations regarding the Cauchy transform along $\Gamma$ can be posed in terms of its parametrized version, which, abusing notation and language, will be again denoted by $C$ and called the Cauchy transform along $\Gamma$. It is defined, for $f \in L^{2}(\mathbf{R})$ and $x \in \mathbf{R}$, by

$$
C f(x)=\text { p.v. } \int_{\mathbf{R}} \frac{f(y)}{z(y)-z(x)} d y .
$$

Associated with it, we consider as well the truncated operators

$$
C_{\epsilon} f(x)=\int_{|y-x|>\epsilon} \frac{f(y)}{z(y)-z(x)} d y
$$

and the maximal operator

$$
C_{*} f(x)=\sup _{\epsilon>0}\left|C_{\epsilon} f(x)\right| .
$$

Notice that the truncated operators $C_{\epsilon}$ are not the exact analogues to the truncated operators $C_{\mu, \epsilon}$ defined above, which would correspond to

$$
\tilde{C}_{\epsilon} f(x)=\int_{|z(y)-z(x)|>\epsilon} \frac{f(y)}{z(y)-z(x)} d y .
$$

We will deal with this issue later.

From the standard Calderón-Zygmund theory, we obtain that $C$ is bounded in $L^{p}(\mathbf{R})$ for $1<p<\infty$, it is bounded from $L^{1}(\mathbf{R})$ to $L^{1, \infty}(\mathbf{R})$ and from $L^{\infty}(\mathbf{R})$ to $B M O(\mathbf{R})$, and it satisfies the classical Cotlar's inequality, i.e., for all $f \in L^{2}(\mathbf{R})$ and all $x \in \mathbf{R}$,

$$
C_{*} f(x) \lesssim M(C f)(x)+C f(x),
$$


where $M$ is the Hardy-Littlewood maximal operator, defined for $h \in L_{\text {loc }}^{1}(\mathbf{R})$ and $x \in \mathbf{R}$ by

$$
M h(x)=\sup _{r>0} \frac{1}{2 r} \int_{x-r}^{x+r}|h(y)| d y .
$$

Here, and throughout the whole paper, the notation $A \lesssim B$ will mean that there exists a constant $c>0$, not depending on $A$ or $B$, such that $A \leq c B$. Also, the notation $A \approx B$ will be equivalent to $A \lesssim B \lesssim A$.

In the papers [7], [8] and [9], Mateu, Orobitg, Pérez and Verdera study the problem of controlling the maximal singular integral $T_{*} f$ in terms of the singular integral $T f$. As it is stated in those papers, one reason to consider this problem is to gain a better understanding of David-Semmes conjecture regarding the possibility of characterizing uniform rectifiability by the boundedness of the Riesz transforms (see $[3])$.

Next we describe some of the results proved in those papers. First, recall that for a Calderón-Zygmund operator with kernel $K=K(x, y)$, the truncated operators $T_{\epsilon}$ are defined, for $\epsilon>0$, by

$$
T_{\epsilon} f(x)=\int_{|y-x|>\epsilon} K(x, y) f(y) d y,
$$

and the associated maximal operator is

$$
T_{*} f(x)=\sup _{\epsilon>0}\left|T_{\epsilon} f(x)\right| .
$$

Definition 1. A higher-order Riesz transform is a Calderón-Zygmund principal value operator defined, for $f \in L^{2}\left(\mathbf{R}^{n}\right)$, by

$$
T f(x)=\text { p.v. } \int_{\mathbf{R}^{n}} \frac{P(x-y)}{|x-y|^{n+d}} f(y) d y,
$$

where $P$ is a harmonic homogeneous polynomial of degree $d \geq 1$. We say that $T$ is odd (respectively, even) if $d$ is odd (respectively, even).

Theorem A. Let $T$ be a higher order Riesz transform, and let $T_{*}$ be the associated maximal operator. Then,

(1) if $T$ is even, then for all $f \in L^{2}\left(\mathbf{R}^{n}\right)$ and all $x \in \mathbf{R}^{n}$,

$$
T_{*} f(x) \lesssim M(T f)(x) .
$$

(2) if $T$ is odd, then for all $f \in L^{2}\left(\mathbf{R}^{n}\right)$ and all $x \in \mathbf{R}^{n}$,

$$
T_{*} f(x) \lesssim M^{2}(T f)(x) .
$$

Definition 2. A smooth homogeneous Calderón-Zygmund operator is a principal value Calderón-Zygmund operator which is defined, for $f \in L^{2}\left(\mathbf{R}^{n}\right)$, by

$$
T f(x)=\text { p.v. } \int_{\mathbf{R}^{n}} \frac{\Omega(x-y)}{|x-y|^{n}} f(y) d y,
$$

where $\Omega: \mathbf{R}^{n} \rightarrow \mathbf{C}$ is a homogeneous function of degree 0 whose restriction to the unit sphere $\mathbf{S}^{n-1}$ is of class $\mathcal{C}^{\infty}$ and satisfies the cancellation property

$$
\int_{\mathbf{S}^{n-1}} \Omega(u) d \sigma(u)=0
$$


where $\sigma$ is the surface measure on $\mathbf{S}^{n-1}$. We will say that the operator is odd (resp., even) if $\Omega$ is odd (resp., even).

Theorem B. Let $T$ be a smooth homogeneous Calderón-Zygmund operator, and let $T_{*}$ be the associated maximal operator. Then,

- if $T$ is even, the following assertions are equivalent:

(1) $T_{*} f(x) \lesssim M(T f)(x)$ for all $f \in L^{2}\left(\mathbf{R}^{n}\right)$ and all $x \in \mathbf{R}^{n}$.

(2) $\left\|T_{*} f\right\|_{L^{2}} \lesssim\|T f\|_{L^{2}}$ for all $f \in L^{2}\left(\mathbf{R}^{n}\right)$.

- if $T$ is odd, the following assertions are equivalent:

(1) $T_{*} f(x) \lesssim M^{2}(T f)(x)$ for all $f \in L^{2}\left(\mathbf{R}^{n}\right)$ and all $x \in \mathbf{R}^{n}$.

(2) $\left\|T_{*} f\right\|_{L^{2}} \lesssim\|T f\|_{L^{2}}$ for all $f \in L^{2}\left(\mathbf{R}^{n}\right)$.

The statements in the previous two theorems concerning even operators were proved in [8], while those concerning odd operators were proved in [7].

Motivated by these results, we consider the problem of controlling the maximal Cauchy transform just in terms of the Cauchy transform. We will only deal with the problem of giving a pointwise estimate of the form

$$
C_{*} f(x) \lesssim M^{n}(C f)(x),
$$

for some $n \in \mathbf{N}, n \geq 2$, since the inequality

$$
\left\|C_{*} f\right\|_{L^{2}(\mathbf{R})} \lesssim\|C f\|_{L^{2}(\mathbf{R})}
$$

is almost trivial, as we will show later (observe that Theorem B cannot be applied to $C$ since it is not a convolution operator).

Notice that the Cauchy transform along a Lipschitz graph $\Gamma$ coincides with a constant multiple of the Hilbert transform when $\Gamma$ is a straight line, and this is a reason why one could think that the pointwise estimate $C_{*} f \lesssim M^{n}(C f)$ could hold for the Cauchy transform along, at least, some class of graphs $\Gamma$. We will show that one cannot have a similar inequality for the Cauchy transform, unless $\Gamma$ is a straight line.

Theorem 1. Consider the Lipschitz function $A(x)=|x|$, and let $C$ denote the Cauchy transform along $\Gamma$, the graph of $A$. Then, there exists $f \in L^{2}(\mathbf{R})$ such that for all $c>0$ and all $n \geq 1$, there exists $\epsilon>0$ such that

$$
\left|C_{\epsilon} f(0)\right|>c M^{n}(C f)(0) \text {. }
$$

This theorem can be easily generalized to Lipschitz graphs $\Gamma$ with angles, meaning with this points $x$ where $A^{\prime}$ has a jump discontinuity, as we will show later.

After obtaining this result, one might think of establishing the inequality $C_{*} f \lesssim$ $M^{n}(C f)$ imposing some restrictions on the smoothness of $A$. This is not the case, as the next theorem shows.

Theorem 2. Let $A$ be a Lipschitz function with compact support, and let $C$ denote the Cauchy transform along $\Gamma$, the graph of $A$. Suppose $A$ is not identically null, or, equivalently, that $\Gamma$ is not a straight line. Then, there exists $x \in \mathbf{R}$ such that for all $c>0$ there exists $f \in L^{2}(\mathbf{R})$ with

$$
C_{*} f(x)>c M^{n}(C f)(x)
$$

for all $n \geq 1$.

We want to remark that the points $x$ mentioned in this last theorem are 'easy' to find. For example, when $A$ is of class $\mathcal{C}^{2}$, any point $x$ with $A^{\prime \prime}(x) \neq 0$ will do the 
job. Notice also that in the case when $A$ has compact support and $\Gamma$ has an angle at a point $x$, the failure of the inequality $C_{*} f(x) \leq c M^{n}(C f)(x)$ for all $f \in L^{2}(\mathbf{R})$ can also be deduced from this result, but Theorem 1 is stronger in this setting, since the argument used there provides a single function $f$ for which the previous inequality fails for all possible constants $c>0$.

\section{Another version of the Cauchy transform}

We define a new operator, which, abusing language, will also be called the Cauchy transform along $\Gamma$, by

$$
T f(x)=\frac{1}{\pi i} \text { p.v. } \int_{\mathbf{R}} \frac{f(y)}{z(y)-z(x)} d z(y),
$$

where $d z(y)=z^{\prime}(y) d y=\left(1+i A^{\prime}(y)\right) d y$. As before, associated with it, we will have the truncated operators $T_{\epsilon}$ and the maximal operator $T_{*}$. This operator is very closely related to $C$. Indeed,

$$
\begin{aligned}
T f(x) & =\frac{1}{\pi i} \text { p.v. } \int_{\mathbf{R}} \frac{f(y)}{z(y)-z(x)} d z(y) \\
& =\frac{1}{\pi i} \text { p.v. } \int_{\mathbf{R}} \frac{f(y) z^{\prime}(y)}{z(y)-z(x)} d y=\frac{1}{\pi i} C\left(f \cdot z^{\prime}\right)(x) .
\end{aligned}
$$

Analogously,

$$
C f(x)=\pi i T\left(\frac{f}{z^{\prime}}\right)(x) .
$$

It is clear that $T$ satisfies the same boundedness properties that $C$ satisfies (with different multiplicative constants). Moreover, by equations (1) and (2), and taking into account that $z^{\prime} \in L^{\infty}$ and $\left|z^{\prime}\right| \approx 1$, we can limit ourselves to prove the Theorems 1 and 2 substituting $C$ by $T, C_{\epsilon}$ by $T_{\epsilon}$ and $C_{*}$ by $T_{*}$.

The main reason for using this version of the Cauchy transform is contained in the following result, which we learnt from Escauriaza [5].

Lemma 1. If $f \in L^{p}(\mathbf{R}), 1<p<\infty$, then $T^{2} f=f$.

Proof. For $w \in \mathbf{C}$ and $\alpha>0$, we define the upper and lower half cones with vertex at $w$ and generatrix slope $\alpha$, respectively, by

$$
\begin{aligned}
& X^{+}(w, \alpha)=\{z \in \mathbf{C}:|\operatorname{Re} z-\operatorname{Re} w|<\alpha(\operatorname{Im} z-\operatorname{Im} w)\} \\
& X^{-}(w, \alpha)=\{z \in \mathbf{C}:|\operatorname{Re} z-\operatorname{Re} w|<\alpha(\operatorname{Im} w-\operatorname{Im} z)\} .
\end{aligned}
$$

It is immediate that for all $w \in \Gamma$ and all $0<\alpha<\frac{1}{\left\|A^{\prime}\right\|_{\infty}}$,

$$
X^{+}(w, \alpha) \subset\{x+i y \in \mathbf{C}: y>A(x)\}
$$

and

$$
X^{-}(w, \alpha) \subset\{x+i y \in \mathbf{C}: y<A(x)\}
$$


Fix $0<\alpha<\frac{1}{\left\|A^{\prime}\right\|_{\infty}}$. Let $f \in L^{p}(\mathbf{R})$, and let us define, for $x \in \mathbf{R}$,

$$
\begin{aligned}
& T_{+} f(x)=\lim _{\substack{w \rightarrow z(x) \\
w \in X^{+}(z(x), \alpha)}} \frac{1}{\pi i} \int_{\mathbf{R}} \frac{f(y)}{w-z(x)} d z(y), \\
& T_{-} f(x)=\lim _{\substack{w \rightarrow z(x) \\
w \in X^{-}(z(x), \alpha)}} \frac{1}{\pi i} \int_{\mathbf{R}} \frac{f(y)}{w-z(x)} d z(y) .
\end{aligned}
$$

From the Plemelj's formulas (see, for example, Chapter 8 of [10]), we obtain

$$
T_{+} f(x)=T f(x)+f(x) ; \quad T_{-} f(x)=T f(x)-f(x)
$$

for a.e. $x \in \mathbf{R}$. In particular, $T=T_{+}-$Id. Hence,

$$
T^{2}=\left(T_{+}-\mathrm{Id}\right)^{2}=\left(T_{+}\right)^{2}-2 T_{+}+\mathrm{Id} .
$$

A direct application of Cauchy's integral formula gives $\left(T_{+}\right)^{2}=2 T_{+}$. As a consequence, $T^{2}=\mathrm{Id}$, as desired.

As a consequence of this result, one easily gets the $L^{p}$ control of the maximal Cauchy transform in terms of the Cauchy transform, for $1<p<\infty$.

Corollary 1. If $f \in L^{p}(\mathbf{R}), 1<p<\infty$, then $\left\|T_{*} f\right\|_{L^{p}} \lesssim\|T f\|_{L^{p}}$.

Proof. Indeed, taking into account the $L^{p}$-boundedness of $T_{*}$ and $T$, and the fact that $T^{2} f=f$, we get

$$
\left\|T^{*} f\right\|_{L^{p}} \lesssim\|f\|_{L^{p}}=\left\|T^{2} f\right\|_{L^{p}} \lesssim\|T f\|_{L^{p}}
$$

The following lemma states that $T$ is antisymmetric with respect to $d z$, and its proof follows by an easy application of Fubini's Theorem.

Lemma 2. Let $1<p<\infty, p^{\prime}$ the conjugate exponent to $p$ and $f \in L^{p}(\mathbf{R})$, $g \in L^{p^{\prime}}(\mathbf{R})$. Then,

$$
\int_{\mathbf{R}} T f(x) g(x) d z(x)=-\int_{\mathbf{R}} f(y) T g(y) d z(y) .
$$

\section{The proofs}

We argue here as Mateu, Orobitg, Pérez and Verdera did in [7], where they proved $T_{*} f \lesssim M^{2}(T f)$, for $T$ an odd higher order Riesz transform.

Let $f \in L^{2}(\mathbf{R}), x \in \mathbf{R}$ and $\epsilon>0$. We have

$$
T_{\epsilon} f(x)=\frac{1}{\pi i} \int_{|y-x|>\epsilon} \frac{f(y)}{z(y)-z(x)} d z(y) .
$$

For $x \in \mathbf{R}$ and $\epsilon>0$, define

$$
K_{x, \epsilon}(y)=\frac{1}{\pi i} \frac{1}{z(y)-z(x)} \chi_{|y-x|>\epsilon}(y),
$$

so that

$$
T_{\epsilon} f(x)=\int_{\mathbf{R}} f(y) K_{x, \epsilon}(y) d z(y) .
$$

A straightforward computation yields that $K_{x, \epsilon} \in L^{2}(\mathbf{R}) \cap L^{\infty}(\mathbf{R})$ and

$$
\left\|K_{x, \epsilon}\right\|_{L^{2}} \leq \frac{1}{\sqrt{\epsilon}}, \quad\left\|K_{x, \epsilon}\right\|_{L^{\infty}} \leq \frac{1}{\epsilon} .
$$


Now let $g_{x, \epsilon}=T\left(K_{x, \epsilon}\right)$, so that

$$
\begin{aligned}
T_{\epsilon} f(x) & =\int_{\mathbf{R}} f(y) K_{x, \epsilon}(y) d z(y)=\int_{\mathbf{R}} f(y) T\left(T\left(K_{x, \epsilon}\right)\right)(y) d z(y) \\
& =-\int_{\mathbf{R}} T f(y) T\left(K_{x, \epsilon}\right)(y) d z(y)=-\int_{\mathbf{R}} T f(y) g_{x, \epsilon}(y) d z(y) .
\end{aligned}
$$

Fix $N>0$ to be chosen later, and denote, for $a \in \mathbf{R}$ and $r>0$,

$$
I_{a, r}=(a-r, a+r) .
$$

Also, for a function $h \in L_{\text {loc }}^{1}(\mathbf{R})$ and an interval $I \subset \mathbf{R}$, denote

$$
m_{I} h=\frac{1}{|I|} \int_{I} h(x) d x
$$

Then, we have,

$$
\begin{aligned}
-T_{\epsilon} f(x)= & \int_{\mathbf{R}} T f(y) g_{x, \epsilon}(y) d z(y) \\
= & \int_{|y-x|<N \epsilon} T f(y) g_{x, \epsilon}(y) d z(y)+\int_{|y-x|>N \epsilon} T f(y) g_{x, \epsilon}(y) d z(y) \\
= & \int_{I_{x, N \epsilon}} T f(y)\left[g_{x, \epsilon}(y)-m_{I_{x, N \epsilon}}\left(g_{x, \epsilon}\right)\right] d z(y)+m_{I_{x, N \epsilon}}\left(g_{x, \epsilon}\right) \int_{I_{x, N \epsilon}} T f(y) d z(y) \\
& +\int_{|y-x|>N \epsilon} T f(y) g_{x, \epsilon}(y) d z(y)=I+I I+I I I .
\end{aligned}
$$

Let us check now that $|I| \lesssim M^{2}(T f)(x)$ and $|I I| \lesssim M(T f)(x)$. We recall first the following tesults, stated in [7], and whose proofs can be found in [11] and [6], respectively:

Lemma 3. Let $\phi \in B M O\left(\mathbf{R}^{n}\right), \psi$ a measurable function in $\mathbf{R}^{n}$ and $Q$ a cube in $\mathbf{R}^{n}$. Then,

$$
\frac{1}{Q} \int_{Q}\left|\phi(x)-m_{Q} \phi\|\psi(x) \mid d x \leq c\| \phi\left\|_{B M O}\right\| \psi \|_{L \log L, Q},\right.
$$

where $c>0$ only depends on $n$.

Lemma 4. There exists a positive constant $c=c(n)>0$ such that for every cube $Q \subset \mathbf{R}^{n}$ and every function $\psi \in L_{\text {loc }}^{1}\left(\mathbf{R}^{n}\right)$ we have

$$
\|\psi\|_{L \log L, Q} \leq c M^{2} \psi(x)
$$

where $M^{2}=M \circ M$ and $M$ is the Hardy-Littlewood maximal operator.

We have

$$
\begin{aligned}
|I| & =\left|\int_{I_{x, N \epsilon}} T f(y)\left[g_{x, \epsilon}(y)-m_{I_{x, N \epsilon}}\left(g_{x, \epsilon}\right)\right] d z(y)\right| \\
& \lesssim \int_{I_{x, N \epsilon}}\left|T f(y) \| g_{x, \epsilon}(y)-m_{I_{x, N \epsilon}}\left(g_{x, \epsilon}\right)\right| d y \\
& \lesssim\left|I_{x, N \epsilon}\right|\left\|g_{x, \epsilon}\right\|_{B M O}\|T f\|_{L(\log L), I_{x, N \epsilon}} \\
& \lesssim \epsilon\left\|T\left(K_{x, \epsilon}\right)\right\|_{B M O} M^{2}(T f)(x) \\
& \lesssim \epsilon\left\|K_{x, \epsilon}\right\|_{L^{\infty}} M^{2}(T f)(x) \lesssim M^{2}(T f)(x) .
\end{aligned}
$$


On the other hand,

$$
\begin{aligned}
|I I| & =\left|m_{I_{x, N \epsilon}}\left(g_{x, \epsilon}\right) \int_{I_{x, N \epsilon}} T f(y) d z(y)\right| \lesssim \frac{1}{\left|I_{x, N \epsilon}\right|}\left|\int_{I_{x, N \epsilon}} g_{x, \epsilon}(y) d y\right| \int_{I_{x, N \epsilon}}|T f(y)| d y \\
& =\left|\int_{I_{x, N \epsilon}} T\left(K_{x, \epsilon}\right)(y) d y\right| \frac{1}{\left|I_{x, N \epsilon}\right|} \int_{I_{x, N \epsilon}}|T f(y)| d y \\
& \leq\left|I_{x, N \epsilon}\right|^{\frac{1}{2}}\left\|T\left(K_{x, \epsilon}\right)\right\|_{L^{2}} M(T f)(x) \lesssim \epsilon^{\frac{1}{2}}\left\|K_{x, \epsilon}\right\|_{L^{2}} M(T f)(x) \lesssim M(T f)(x),
\end{aligned}
$$

as claimed.

Now, since $M(T f) \leq M^{2}(T f)$, we get

$$
|I|+|I I| \lesssim M^{2}(T f)(x)
$$

Let us study III now. Recall that

$$
I I I=\int_{|y-x|>N \epsilon} T f(y) g_{x, \epsilon}(y) d z(y)=\int_{|y-x|>N \epsilon} T f(y) T\left(K_{x, \epsilon}(y)\right) d z(y) .
$$

An easy contour integration argument yields the following result:

Lemma 5. Fix $x \in \mathbf{R}$ and $\epsilon>0$. Then, for almost every $y \in \mathbf{R}$ with $|y-x|>\epsilon$, we have

$$
T\left(K_{x, \epsilon}\right)(y)=\frac{1}{\pi i} \frac{1}{z(y)-z(x)}\left[B(x, \epsilon)+G_{x, \epsilon}(y)\right]
$$

where

$$
B(x, \epsilon)=\log \frac{|z(x+\epsilon)-z(x)|}{|z(x-\epsilon)-z(x)|}+i(\pi+\arg [z(x+\epsilon)-z(x)]-\arg [z(x-\epsilon)-z(x)])
$$

and

$$
G_{x, \epsilon}(y)=\log \frac{|z(x-\epsilon)-z(y)|}{|z(x+\epsilon)-z(y)|}+i(\arg [z(x-\epsilon)-z(y)]-\arg [z(x+\epsilon)-z(y)]),
$$

where, for a complex number $w \neq 0$, we consider $-\frac{\pi}{2} \leq \arg (w)<\frac{3 \pi}{2}$.

Proof. Let $x \in \mathbf{R}, \epsilon>0$ and $y \in \mathbf{R}$ with $|y-x|>\epsilon$. We will assume that $y>x$ (the case $y<x$ is treated analogously) and also that $A$ is differentiable at $y$. For a set $I \subset \mathbf{R}$, denote $\Gamma(I)=\{z(t): t \in I\}$, and for a complex number $w \neq 0$, let $\log (w)=\log |w|+i \arg (w)$. Then, we have

$$
\begin{aligned}
& T\left(K_{x, \epsilon}\right)(y)=\frac{1}{\pi i} \text { p.v. } \int_{\mathbf{R}} \frac{K_{x, \epsilon}(t)}{z(t)-z(y)} d z(t) \\
& =\lim _{\substack{R \rightarrow \infty \\
\delta \rightarrow 0}} \frac{1}{\pi i} \int_{\Gamma(\{t:|t-x|>\epsilon,|t-y|>\delta,|t|<R\})} \frac{d w}{(w-z(x))(w-z(y))} \\
& =\frac{1}{\pi i(z(y)-z(x))} \lim _{\substack{R \rightarrow \infty \\
\delta \rightarrow 0}} \int_{\Gamma(\{t:|t-x|>\epsilon,|t-y|>\delta,|t|<R\})}\left(\frac{1}{w-z(y)}-\frac{1}{w-z(x)}\right) d w \\
& =\frac{1}{\pi i(z(y)-z(x))} \lim _{\substack{R \rightarrow \infty \\
\delta \rightarrow 0}}\left(I_{R, \delta}+I I_{R, \delta}+I I I_{R, \delta}\right),
\end{aligned}
$$


where, for sufficiently small $\delta>0$ and sufficiently big $R>0$,

$$
\begin{aligned}
I_{R, \delta}= & \int_{\Gamma((-R, x-\epsilon))}\left(\frac{1}{w-z(y)}-\frac{1}{w-z(x)}\right) d w \\
= & \log [z(x-\epsilon)-z(y)]-\log [z(-R)-z(y)]-\log [z(x-\epsilon)-z(x)] \\
& +\log [z(-R)-z(x)], \\
I I_{R, \delta}= & \int_{\Gamma((x+\epsilon, y-\delta))}\left(\frac{1}{w-z(y)}-\frac{1}{w-z(x)}\right) d w \\
= & \log [z(y-\delta)-z(y)]-\log [z(x+\epsilon)-z(y)]-\log [z(y-\delta)-z(x)] \\
& +\log [z(x+\epsilon)-z(x)]
\end{aligned}
$$

and

$$
\begin{aligned}
I I I_{R, \delta}= & \int_{\Gamma((y+\delta, R))}\left(\frac{1}{w-z(y)}-\frac{1}{w-z(x)}\right) d w \\
= & \log [z(R)-z(y)]-\log [z(y+\delta)-z(y)]-\log [z(R)-z(x)] \\
& +\log [z(y+\delta)-z(x)] .
\end{aligned}
$$

Gathering the previous identities, we obtain

$$
\begin{aligned}
\operatorname{Re}\left(I_{R, \delta}+I I_{R, \delta}+I I I_{R, \delta}\right)= & \log \frac{|z(x-\epsilon)-z(y)||z(x+\epsilon)-z(x)|}{|z(x+\epsilon)-z(y)||z(x-\epsilon)-z(x)|} \\
& +\log \frac{|z(-R)-z(x)||z(R)-z(y)|}{|z(-R)-z(y)||z(R)-z(x)|} \\
& +\log \frac{|z(y-\delta)-z(y)||z(y+\delta)-z(x)|}{|z(y+\delta)-z(y)||z(y-\delta)-z(x)|} .
\end{aligned}
$$

On the other hand,

$$
\begin{aligned}
\operatorname{Im}\left(I_{R, \delta}+I I_{R, \delta}+I I I_{R, \delta}\right)= & (\arg [z(x-\epsilon)-z(y)]-\arg [z(x-\epsilon)-z(x)] \\
& -\arg [z(x+\epsilon)-z(y)]+\arg [z(x+\epsilon)-z(x)]) \\
& +(-\arg [z(-R)-z(y)]+\arg [z(-R)-z(x)] \\
& +\arg [z(R)-z(y)]-\arg [z(R)-z(x)]) \\
& +(\arg [z(y-\delta)-z(y)]-\arg [z(y-\delta)-z(x)] \\
& -\arg [z(y+\delta)-z(y)]+\arg [z(y+\delta)-z(x)]) .
\end{aligned}
$$

Gathering the identities (5) and (6), letting $R \rightarrow \infty$ and $\delta \rightarrow 0$, and using the fact that $A$ is differentiable at $y$, we obtain

$$
\lim _{\substack{R \rightarrow \infty \\ \delta \rightarrow 0}}\left(I_{R, \delta}+I I_{R, \delta}+I I I_{R, \delta}\right)=G_{x, \epsilon}(y)+B(x, \epsilon),
$$

and so the desired conclusion follows.

It is easy to check that the term $B(x, \epsilon)$ satisfies the following:

Lemma 6. Let $x \in \mathbf{R}$ and $\epsilon>0$. Then, the following assertions are equivalent:

(1) $B(x, \epsilon)=0$.

(2) $\operatorname{Im} B(x, \epsilon)=0$.

(3) The points $z(x-\epsilon), z(x)$ and $z(x+\epsilon)$ are collinear. 
On the other hand, we can prove the following decay at infinity of the term $G_{x, \epsilon}(y)$.

Lemma 7. Choose $N>1+4\left(1+\left\|A^{\prime}\right\|_{\infty}\right)$. Then for $|y-x|>N \epsilon$,

$$
\left|G_{x, \epsilon}(y)\right| \lesssim \frac{\epsilon}{|y-x|}
$$

Proof. Denote $\Lambda_{1}=\left\|A^{\prime}\right\|_{\infty}$, and let

$$
u_{x, \epsilon}(y)=\operatorname{Re} G_{x, \epsilon}(y)=\log \frac{|z(x-\epsilon)-z(y)|}{|z(x+\epsilon)-z(y)|}
$$

and

$$
v_{x, \epsilon}(y)=\operatorname{Im} G_{x, \epsilon}(y)=\arg [z(x-\epsilon)-z(y)]-\arg [z(x+\epsilon)-z(y)] .
$$

Recall that, for $w \in \mathbf{C},|w|<\frac{1}{2}$,

$$
|\log (1+w)| \leq 2|w|,
$$

where Log is defined as in the previous lemma.

Now, for $|y-x|>N \epsilon$, we have

$$
\frac{z(x-\epsilon)-z(y)}{z(x+\epsilon)-z(y)}=1+\frac{z(x-\epsilon)-z(x+\epsilon)}{z(x+\epsilon)-z(y)},
$$

and

$$
\begin{aligned}
\left|\frac{z(x-\epsilon)-z(x+\epsilon)}{z(x+\epsilon)-z(y)}\right| & \leq \frac{\left(1+\Lambda_{1}\right) 2 \epsilon}{|y-(x+\epsilon)|} \leq \frac{\left(1+\Lambda_{1}\right) 2 \epsilon}{\frac{N-1}{N}|y-x|} \\
& \leq \frac{\left(1+\Lambda_{1}\right) 2 \epsilon}{\frac{N-1}{N} N \epsilon}=\frac{2\left(1+\Lambda_{1}\right)}{N-1} \leq \frac{1}{2}
\end{aligned}
$$

where the last inequality holds precisely because of the choice of $N$. Then,

$$
\begin{aligned}
\left|u_{x, \epsilon}(y)\right| & =\left|\log \frac{|z(x-\epsilon)-z(y)|}{|z(x+\epsilon)-z(y)|}\right|=|\log | 1+\frac{z(x-\epsilon)-z(x+\epsilon)}{z(x+\epsilon)-z(y)}|| \\
& \leq\left|\log \left(1+\frac{z(x-\epsilon)-z(x+\epsilon)}{z(x+\epsilon)-z(y)}\right)\right| \leq 2\left|\frac{z(x-\epsilon)-z(x+\epsilon)}{z(x+\epsilon)-z(y)}\right| \\
& \leq 2 \frac{\left(1+\Lambda_{1}\right) 2 \epsilon}{\frac{N-1}{N}|y-x|}=\frac{4 N\left(1+\Lambda_{1}\right)}{N-1} \frac{\epsilon}{|y-x|} \lesssim \frac{\epsilon}{|y-x|} .
\end{aligned}
$$

On the other hand,

$$
\begin{aligned}
\left|v_{x, \epsilon}(y)\right| & =|\arg [z(x-\epsilon)-z(y)]-\arg [z(x+\epsilon)-z(y)]| \\
& \leq|\log [z(x-\epsilon)-z(y)]-\log [z(x+\epsilon)-z(y)]| \\
& =\left|\int_{\Gamma((x-\epsilon, x+\epsilon))} \frac{d z}{z-z(y)}\right| \leq \operatorname{length}(\Gamma((x-\epsilon, x+\epsilon))) \max _{|t-x| \leq \epsilon} \frac{1}{|z(t)-z(y)|} \\
& \leq \frac{2\left(1+\Lambda_{1}\right) \epsilon}{|y-x|} \lesssim \frac{\epsilon}{|y-x|} .
\end{aligned}
$$

Putting all together, the lemma follows. 
As a result, going back to (4), and applying Lemma 5, we obtain

$$
\begin{aligned}
I I I & =\frac{1}{\pi i} \int_{|y-x|>N \epsilon} T f(y) \frac{1}{z(y)-z(x)}\left[B(x, \epsilon)+G_{x, \epsilon}(y)\right] d z(y) \\
& =\frac{1}{\pi i}\left[B(x, \epsilon) \int_{|y-x|>N \epsilon} T f(y) \frac{d z(y)}{z(y)-z(x)}+\int_{|y-x|>N \epsilon} T f(y) \frac{G_{x, \epsilon}(y) d z(y)}{z(y)-z(x)}\right] \\
& =B(x, \epsilon) T_{N \epsilon}(T f)(x)+I V .
\end{aligned}
$$

Now, fixing $N>1+4\left(1+\left\|A^{\prime}\right\|_{\infty}\right)$, and applying Lemma 7 , we obtain

$$
\begin{aligned}
|I V| & =\left|\frac{1}{\pi i} \int_{|y-x|>N \epsilon} T f(y) \frac{G_{x, \epsilon}(y) d z(y)}{z(y)-z(x)}\right| \lesssim \epsilon \int_{|y-x|>N \epsilon}|T f(y)| \frac{d y}{|y-x|^{2}} \\
& =\epsilon \sum_{k=0}^{\infty} \int_{2^{k} N \epsilon<|y-x|<2^{k+1} N \epsilon}|T f(y)| \frac{d y}{|y-x|^{2}} \\
& \leq \epsilon \sum_{k=0}^{\infty} \frac{1}{\left(2^{k} N \epsilon\right)^{2}} \int_{2^{k} N \epsilon<|y-x|<2^{k+1} N \epsilon}|T f(y)| d y \\
& \leq \epsilon \sum_{k=0}^{\infty} \frac{1}{2^{k-2} N \epsilon} \frac{1}{2 \cdot 2^{k+1} N \epsilon} \int_{|y-x|<2^{k+1} N \epsilon} \\
& \leq \epsilon\left(\sum_{k=0}^{\infty} \frac{1}{2^{k-2} N \epsilon}\right) M(T f)(x) \lesssim M(T f)(x) \leq M^{2}(T f)(x) .
\end{aligned}
$$

As a result, gathering the estimates in (3), (7) and (8), we have the following:

Lemma 8. For all $f \in L^{2}(\mathbf{R})$, all $x \in \mathbf{R}$ and all $\epsilon>0$,

$$
\left|T_{\epsilon} f(x)+B(x, \epsilon) T_{N \epsilon}(T f)(x)\right| \lesssim M^{2}(T f)(x) .
$$

3.1. Proof of Theorem 1. Fix the Lipschitz function $A(x)=|x|$. In this case,

$$
B(0, \epsilon)=\log \frac{|z(\epsilon)-z(0)|}{|z(-\epsilon)-z(0)|}+i(\pi+\arg [z(\epsilon)-z(0)]-\arg [z(-\epsilon)-z(0)])=\frac{\pi i}{2} .
$$

Assume that the inequality

$$
T_{*} f(x) \lesssim M^{n}(T f)(x) \text { for all } f \in L^{2}(\mathbf{R})
$$

were true for some $n \geq 2$. Then, applying Lemma 8 , this would yield

$$
\left|B(x, \epsilon) T_{N \epsilon}(T f)(x)\right| \lesssim M^{n}(T f)(x)
$$

for all $f \in L^{2}(\mathbf{R})$. Now, taking into account that $T^{2}=\mathrm{Id}$, and setting $x=0$, the latter implies

$$
\left|T_{N \epsilon} f(0)\right| \lesssim M^{n} f(0)
$$

for all $f \in L^{2}(\mathbf{R})$, and this is false for $f=\chi_{[0,1]}$. Indeed, $M^{n} f(0) \leq 1$, while for $0<N \epsilon<1$,

$$
\begin{aligned}
T_{N \epsilon} f(0) & =\frac{1}{\pi i} \int_{|y|>N \epsilon} \chi_{[0,1]}(y) \frac{d z(y)}{z(y)-z(0)}=\frac{1}{\pi i} \int_{N \epsilon}^{1} \frac{1+i}{y+i y} d y \\
& =\frac{1}{\pi i} \int_{N \epsilon}^{1} \frac{d y}{y}=-\frac{1}{\pi i} \log (N \epsilon)
\end{aligned}
$$


SO

$$
\lim _{\epsilon \rightarrow 0}\left|T_{N \epsilon} f(0)\right|=\infty
$$

yielding a contradiction with (9).

This counterexample can be generalized in the following way. Suppose $\Gamma$ has an angle at a point $z(x), x \in \mathbf{R}$, meaning with this that $A^{\prime}$ has a jump discontinuity at $x$, i.e.,

$$
\lim _{h \rightarrow 0^{+}} \frac{A(x+h)-A(x)}{h}=A_{+}^{\prime}(x) \neq A_{-}^{\prime}(x)=\lim _{h \rightarrow 0^{-}} \frac{A(x+h)-A(x)}{h} .
$$

A straightforward computation shows now that

$$
\lim _{\epsilon \rightarrow 0} \operatorname{Im} B(x, \epsilon)=\arctan \left(A_{+}^{\prime}(x)\right)-\arctan \left(A_{-}^{\prime}(x)\right) \neq 0,
$$

and so $B(x, \epsilon)$ stays away from 0 as $\epsilon \rightarrow 0$. The same argument that was used above, substituting $\chi_{[0,1]}$ by $\chi_{[x, x+1]}$, will show that the inequality

$$
T_{*} f(x) \lesssim M^{n}(T f)(x)
$$

cannot hold.

3.2. Proof of Theorem 2. We will study now the term $T_{N \epsilon}(T f)(x)$ to give more light to this subject. This will lead us to prove that, when $A$ has compact support, the inequality

$$
T_{*} f(x) \lesssim M^{n}(T f)(x)
$$

can only hold when $A=0$, i.e., when $\Gamma$ is a straight line, which is a case already known since $T$ is, essentialy, the Hilbert transform.

Assume that $A$ has compact support, $\operatorname{say} \operatorname{supp}(A) \subset[-L, L], L>0$. Let $f \in$ $L^{2}(\mathbf{R})$, and write

$$
g=(T f) \chi_{[-2 L, 2 L]}, \quad h=(T f) \chi_{\mathbf{R} \backslash[-2 L, 2 L]},
$$

so that $T f=g+h$ and

$$
T_{N \epsilon}(T f)(x)=T_{N \epsilon} g(x)+T_{N \epsilon} h(x) .
$$

Fix $x \in[-L, L]$. Observe that

$$
i \pi T_{N \epsilon} g(x)=\int_{|y-x|>N \epsilon} \frac{g(y)}{z(y)-z(x)} d z(y)=\sum_{k=0}^{\infty} \int_{2^{k}{ } \epsilon<|y-x|<2^{k+1} N \epsilon} \frac{g(y)}{z(y)-z(x)} d z(y) .
$$

Now, taking into account that $\operatorname{supp}(g) \subset[-2 L, 2 L]$, one gets that, when $2^{k} N \epsilon>$ $4 L$,

$$
\int_{2^{k} N \epsilon<|y-x|<2^{k+1} N \epsilon} \frac{g(y)}{z(y)-z(x)} d z(y)=0 .
$$

This yields that only the first $M_{L, \epsilon}$ terms of the sum above do not vanish, where

$$
M_{L, \epsilon}=\left\lceil\frac{\log \left(\frac{4 L}{N \epsilon}\right)}{\log 2}\right\rceil
$$

(by $\lceil t\rceil$ we denote the smallest integer $n$ such that $t \leq n$ ). 
Furthermore, for each $k \geq 0$,

$$
\begin{aligned}
\left|\int_{2^{k} N \epsilon<|y-x|<2^{k+1} N \epsilon} \frac{g(y)}{z(y)-z(x)} d z(y)\right| & \lesssim \int_{2^{k} N \epsilon<|y-x|<2^{k+1} N \epsilon} \frac{|g(y)|}{|y-x|} d y \\
& \lesssim \frac{1}{2^{k} N \epsilon} \int_{|y-x|<2^{k+1} N \epsilon}|g(y)| d y \lesssim M g(x) .
\end{aligned}
$$

Putting all together, and taking into account that $M g \leq M(T f)$, we obtain

$$
\left|T_{N \epsilon} g(x)\right| \lesssim\left(1+\left|\frac{\log \left(\frac{4 L}{N \epsilon}\right)}{\log 2}\right|\right) M(T f)(x) .
$$

On the other hand, since $A=0$ on $\operatorname{supp}(h)$, we get

$$
i \pi T_{N \epsilon} h(x)=\int_{|y-x|>N \epsilon} \frac{h(y)}{z(y)-z(x)} d z(y)=\int_{|y-x|>N \epsilon} \frac{h(y)}{y-z(x)} d y .
$$

Now, for $|y-x|>N \epsilon$,

$$
\frac{1}{y-z(x)}=\frac{1}{y-x}+\left(\frac{1}{y-z(x)}-\frac{1}{y-x}\right)=\frac{1}{y-x}+D(x, y)
$$

and so

$$
\begin{aligned}
i \pi T_{N \epsilon} h(x) & =\int_{|y-x|>N \epsilon} \frac{h(y)}{y-x} d y+\int_{|y-x|>N \epsilon} h(y) D(x, y) d y \\
& :=H_{N \epsilon} h(x)+\int_{|y-x|>N \epsilon} h(y) D(x, y) d y .
\end{aligned}
$$

Observe now that, for $x \neq y$,

$$
|D(x, y)|=\left|\frac{1}{y-z(x)}-\frac{1}{y-x}\right|=\left|\frac{i A(x)}{(y-x)(y-z(x))}\right| \leq \frac{|A(x)|}{|y-x|^{2}} .
$$

Then, taking into account that $h=0$ on $[-2 L, 2 L]$, and recalling that $|x| \leq L$, one gets

$$
\left|\int_{|y-x|>N \epsilon} h(y) D(x, y) d y\right| \leq|A(x)| \int_{|y-x|>L} \frac{|h(y)|}{|y-x|^{2}} d y .
$$

Splitting the last integral into the regions $\left\{2^{k} L<|y-x| \leq 2^{k+1} L\right\}$, and using the fact that $M(h) \leq M(T f)$, we get

$$
\left|\int_{|y-x|>N \epsilon} h(y) D(x, y) d y\right| \leq \frac{8}{L}|A(x)| M(T f)(x) .
$$

The previous discussion shows that

$$
T_{N \epsilon}(T f)(x)=\frac{1}{\pi i} H_{N \epsilon} h(x)+V,
$$

where

$$
|V| \leq c(x, \epsilon, N, L) M(T f)(x)
$$

and $0<c(x, \epsilon, N, L)<\infty$. Recall now that, by Lemma 8 , we have

$$
\left|T_{\epsilon} f(x)+B(x, \epsilon) T_{N \epsilon}(T f)(x)\right| \lesssim M^{2}(T f)(x) .
$$


Then, it follows that

$$
\left|T_{\epsilon} f(x)+\frac{1}{\pi i} B(x, \epsilon) H_{N \epsilon} h(x)\right| \leq c^{\prime}(x, \epsilon, N, L) M^{2}(T f)(x),
$$

where $0<c^{\prime}(x, \epsilon, N, L)<\infty$.

Assume $A$ is not identically null, and suppose that the inequality $T_{*} f(x) \lesssim$ $M^{n}(T f)(x)$ holds. Applying Lemma 6, we may pick $x \in[-L, L]$ and $\epsilon>0$ with

$$
-L<x-N \epsilon<x<x+N \epsilon<L
$$

and such that $B(x, \epsilon) \neq 0$. Then, it follows that

$$
|B(x, \epsilon)|\left|H_{N \epsilon}\left((T f) \chi_{\mathbf{R} \backslash[-2 L, 2 L]}\right)(x)\right| \leq c^{\prime \prime}(x, \epsilon, N, L) M^{n}(T f)(x),
$$

with $0<c^{\prime \prime}(x, \epsilon, N, L)<\infty$.

Now, for each $k=3,4, \ldots$, pick $f_{k} \in L^{2}(\mathbf{R})$ such that $T f_{k}=\chi_{[0, k L]}$, and so $\left(T f_{k}\right) \chi_{\mathbf{R} \backslash[-2 L, 2 L]}=\chi_{(2 L, k L]}$. Applying the previous inequality for each $f_{k}$, and using that $M^{n}\left(T f_{k}\right) \leq 1$, we obtain

$$
|B(x, \epsilon)|\left|H_{N \epsilon}\left(\chi_{(2 L, k L]}\right)(x)\right| \leq c^{\prime \prime}(x, \epsilon, N, L) .
$$

Finally, observe that

$$
H_{N \epsilon}\left(\chi_{(2 L, k L]}\right)(x)=\int_{2 L}^{k L} \frac{d y}{y-x}=\log \frac{k L-x}{2 L-x},
$$

and so

$$
|B(x, \epsilon)| \log \frac{k L-x}{2 L-x} \leq c^{\prime \prime}(x, \epsilon, N, L),
$$

yielding a contradiction, since the left hand side tends to $\infty$ as $k \rightarrow \infty$.

\section{Further results}

4.1. Another version of the truncated operators. Let us consider now another version of the truncated operators. Define, for $\epsilon>0$ and $x \in \mathbf{R}$,

$$
\tilde{T}_{\epsilon} f(x)=\frac{1}{\pi i} \int_{|z(y)-z(x)|>\epsilon} \frac{f(y)}{z(y)-z(x)} d z(y)
$$

and the associated maximal operator $\tilde{T}_{*} f(x)=\sup _{\epsilon>0}\left|\tilde{T}_{\epsilon} f(x)\right|$. This is a truncation over balls of radius $\epsilon$, while the one for $T_{\epsilon}$ was a truncation over strips of width $2 \epsilon$. form

We consider now the same problem as before: that of giving an estimate of the

$$
\tilde{T}_{*} f(x) \lesssim M^{n}(T f)(x),
$$

and the same arguments employed before will work here. Indeed, if we define $l(x, \epsilon)=$ $z\left(x_{-}\right), r(x, \epsilon)=z\left(x_{+}\right)$, where

$$
x_{-}=\sup \{t<x:|z(t)-z(x)|=\epsilon\}
$$

and

$$
x_{+}=\inf \{t>x:|z(t)-z(x)|=\epsilon\},
$$

then $l(x, \epsilon)$ and $r(x, \epsilon)$ will play the same role that $z(x-\epsilon)$ and $z(x+\epsilon)$ played before. Precisely, $l(x, \epsilon)$ is the last point of $\Gamma$ to the left of $z(x)$ that belongs to the circle centered at $z(x)$ with radius $\epsilon$, and $r(x, \epsilon)$ is the analogue of this one at the right. 
Since the quantities $|y-x|$ and $|z(y)-z(x)|$ are comparable, one can repeat the arguments used before to get an analogous of Lemma 8, which will be stated now as

$$
\left|\tilde{T}_{\epsilon} f(x)+\tilde{B}(x, \epsilon) \tilde{T}_{N \epsilon} f(x)\right| \lesssim M^{2}(T f)(x),
$$

where

$$
\tilde{B}(x, \epsilon)=\log \frac{|r(x, \epsilon)-z(x)|}{|l(x, \epsilon)-z(x)|}+i(\pi+\arg [r(x, \epsilon)-z(x)]-\arg [l(x, \epsilon)-z(x)]) .
$$

As in Lemma $6, \tilde{B}(x, \epsilon)=0$ if, and only if, $l(x, \epsilon), z(x)$ and $r(x, \epsilon)$ are collinear.

With this tools at hand, one can prove the following results, which are the analogs to Theorems 1 and 2 in this setting.

Theorem 3. Consider the Lipschitz function $A(x)=|x|$. Then, there exists $f \in L^{2}(\mathbf{R})$ such that for all $c>0$ and all $n \geq 1$, there exists $\epsilon>0$ such that

$$
\left|\tilde{T}_{\epsilon} f(0)\right|>c M^{n}(T f)(0)
$$

To prove this, one can mimic the argument in section 3.1, since here we have again $\tilde{B}(0, \epsilon)=i \frac{\pi}{2}$.

Theorem 4. Let $A$ be a Lipschitz function with compact support. Suppose $A$ is not identically null, or, equivalently, that $\Gamma$ is not a straight line. Then, there exists $x \in \mathbf{R}$ such that for all $c>0$ there exists $f \in L^{2}(\mathbf{R})$ with

$$
\tilde{T}_{*} f(x)>c \tilde{T}^{n}(T f)(x)
$$

for all $n \geq 1$.

Again, the argument in Section 3.2 adapts trivially to this case, by just taking into account that, if $A$ is not identically null, one can find $x \in \mathbf{R}$ and $\epsilon>0$ as small as needed such that $l(x, \epsilon), z(x)$ and $r(x, \epsilon)$ are not collinear.

4.2. The case of Jordan curves. Let $\Gamma$ be a Jordan curve in the plane, parametrized by a periodic function $\gamma: \mathbf{R} \rightarrow \mathbf{C}$. We will pose, for the moment, the following assumptions on $\gamma$ :

- $\gamma$ is of class $\mathcal{C}^{1}$.

- $\gamma$ is $L$-periodic, $\gamma([0, L))=\Gamma$.

- $\gamma$ is injective on $[0, L)$.

- $\left|\gamma^{\prime}(t)\right|=1$ for all $t$.

- $\omega$ is the modulus of continuity of $\gamma^{\prime}$ (this means that $\omega$ is a non-negative and increasing continuous function in $[0, \infty)$ with $\omega(0)=0$ and such that $\left|\gamma^{\prime}(s)-\gamma^{\prime}(t)\right| \leq \omega(|s-t|)$ for all $\left.s, t \in \mathbf{R}\right)$.

We denote by $\mu$ the arc-length measure on $\Gamma$. We have, for a Borel set $I \subset[0, L)$,

$$
\mu(\gamma(I))=\int_{I}\left|\gamma^{\prime}(t)\right| d t=|I| .
$$

For a point $z \in \Gamma$ and $r>0$, denote

$$
\Gamma_{z, r}=\gamma(\{t:|t-x|<r\}),
$$

where $z=\gamma(x), x \in \mathbf{R}$. 
The Hardy-Littlewood maximal function of a function $f \in L^{1}(\Gamma, \mu)$ is defined, for $z \in \Gamma$, by

$$
M f(z)=\sup _{r>0} \frac{1}{\mu\left(\Gamma_{z, r}\right)} \int_{\Gamma_{z, r}}|f| d \mu=\sup _{r>0} \frac{1}{2 r} \int_{\Gamma_{z, r}}|f| d \mu .
$$

The Cauchy transform of a function $f \in L^{2}(\Gamma, d \mu)$ is defined, for $z \in \Gamma$, as the principal value integral

$$
T f(z)=\lim _{\epsilon \rightarrow 0} T_{\epsilon} f(z)
$$

where

$$
T_{\epsilon} f(z)=\frac{1}{\pi i} \int_{\Gamma \backslash \Gamma_{z, \epsilon}} \frac{f(\xi)}{\xi-z} d \xi
$$

We consider as well the maximal operator associated with $T$,

$$
T_{*} f(z)=\sup _{\epsilon>0}\left|T_{\epsilon} f(z)\right| .
$$

In this section we will prove that, if $\gamma$ is regular enough (we will specify later how much regularity is needed), then

$$
T_{*} f(z) \lesssim M^{2}(T f)(z) \text { for all } f \in L^{2}(\Gamma, \mu)
$$

We will follow, essentially, the same steps we have taken in Section 3 for the case of Lipschitz graphs. Most of the arguments there will be valid in this setting, and so we will not enter into many details. First of all, we remark that the analogues of Lemmas 1 and 2 hold now:

Lemma 9. If $f \in L^{2}(\Gamma, \mu), T^{2} f=f$.

Lemma 10. If $f, g \in L^{2}(\Gamma, \mu)$, then

$$
\int_{\Gamma} T f(z) g(z) d z=-\int_{\Gamma} f(z) T g(z) d z .
$$

We argue now as in Section 3. Fix $f \in L^{2}(\Gamma, \mu), z \in \Gamma$ and $\epsilon>0$. Then, we have

$$
T_{\epsilon} f(z)=\frac{1}{\pi i} \int_{\Gamma \backslash \Gamma_{z, \epsilon}} \frac{f(\xi)}{\xi-z} d \xi=\int_{\Gamma} f(\xi) K_{z, \epsilon}(\xi) d \xi,
$$

where

$$
K_{z, \epsilon}(\xi)=\frac{1}{\pi i(\xi-z)} \chi_{\Gamma \backslash \Gamma_{z, \epsilon}}(\xi) .
$$

It is easy to check that $K_{z, \epsilon} \in L^{2}(\Gamma, \mu) \cap L^{\infty}(\Gamma, \mu)$, and moreover

$$
\left\|K_{z, \epsilon}\right\|_{L^{2}} \lesssim \frac{1}{\sqrt{\epsilon}}, \quad\left\|K_{z, \epsilon}\right\|_{L^{\infty}} \lesssim \frac{1}{\epsilon}
$$

Since $K_{z, \epsilon} \in L^{2}(\Gamma, \mu)$, we have $K_{z, \epsilon}=T^{2}\left(K_{z, \epsilon}\right)=T\left(g_{z, \epsilon}\right)$, for $g_{z, \epsilon}=T\left(K_{z, \epsilon}\right)$. Then, we get

$$
T_{\epsilon} f(z)=\int_{\Gamma} f(\xi) K_{z, \epsilon}(\xi) d \xi=\int_{\Gamma} f(\xi) T\left(g_{z, \epsilon}\right)(\xi) d \xi=-\int_{\Gamma} T f(\xi) g_{z, \epsilon}(\xi) d \xi,
$$


and, as a consequence,

$$
\begin{aligned}
-T_{\epsilon} f(z)= & \int_{\Gamma} T f(\xi) g_{z, \epsilon}(\xi) d \xi=\int_{\Gamma_{z, 2 \epsilon}} T f(\xi) g_{z, \epsilon}(\xi) d \xi+\int_{\Gamma \backslash \Gamma_{z, 2 \epsilon}} T f(\xi) g_{z, \epsilon}(\xi) d \xi \\
= & \int_{\Gamma_{z, 2 \epsilon}} T f(\xi)\left[g_{z, \epsilon}(\xi)-m_{\Gamma_{z, 2 \epsilon}}\left(g_{z, \epsilon}\right)\right] d \xi+m_{\Gamma_{z, 2 \epsilon}}\left(g_{z, \epsilon}\right) \int_{\Gamma_{z, 2 \epsilon}} T f(\xi) d \xi \\
& +\int_{\Gamma \backslash \Gamma_{z, 2 \epsilon}} T f(\xi) g_{z, \epsilon}(\xi) d \xi=I+I I+I I I,
\end{aligned}
$$

where, for a function $h \in L^{1}(\Gamma, \mu)$ and a Borel set $E \subset \Gamma$ with $\mu(E)>0$,

$$
m_{E} h=\frac{1}{\mu(E)} \int_{E} h d \mu
$$

Arguing essentially as in Section 3, one can prove that $|I| \lesssim M^{2}(T f)(z)$ and $|I I| \lesssim M(T f)(z)$. Let us study $I I I$ now,

$$
I I I=\int_{\Gamma \backslash \Gamma_{z, 2 \epsilon}} T f(\xi) g_{z, \epsilon}(\xi) d \xi=\int_{\Gamma \backslash \Gamma_{z, 2 \epsilon}} T f(\xi) T\left(K_{z, \epsilon}\right)(\xi) d \xi .
$$

A similar argument to the one used in Lemma 5 yields the following result.

Lemma 11. For $\xi \in \Gamma \backslash \Gamma_{z, 2 \epsilon}$,

$$
T\left(K_{z, \epsilon}\right)(\xi)=\frac{1}{\pi i} \frac{1}{z-\xi}\left[B(z, \epsilon)+G_{z, \epsilon}(\xi)\right]
$$

where

$$
G_{z, \epsilon}(\xi) \lesssim \frac{\epsilon}{|z-\xi|}
$$

and

$$
|B(z, \epsilon)| \lesssim \omega(2 \epsilon)
$$

Remark. The expressions of $G_{z, \epsilon}(\xi)$ and $B(z, \epsilon)$ are totally analogous to the ones for $G_{x, \epsilon}(y)$ and $B(x, \epsilon)$ in Lemma 5 , for suitably chosen branches of $\arg (w-z)$ and $\arg (w-\xi)$. The estimate for $G_{z, \epsilon}$ is proved as in Lemma 7, while the estimate for $B(z, \epsilon)$ follows from an application of the Mean Value Theorem.

From this, it follows that

$$
\begin{aligned}
I I I & =\int_{\Gamma \backslash \Gamma_{z, 2 \epsilon}} T f(\xi) T\left(K_{z, \epsilon}\right)(\xi) d \xi \\
& =B(z, \epsilon) \frac{1}{\pi i} \int_{\Gamma \backslash \Gamma_{z, 2 \epsilon}} T f(\xi) \frac{1}{z-\xi} d \xi+\frac{1}{\pi i} \int_{\Gamma \backslash \Gamma_{z, 2 \epsilon}} T f(\xi) \frac{G_{z, \epsilon}(\xi)}{z-\xi} d \xi \\
& =B(z, \epsilon) T_{2 \epsilon}(T f)(z)+\frac{1}{\pi i} \int_{\Gamma \backslash \Gamma_{z, 2 \epsilon}} T f(\xi) \frac{G_{z, \epsilon}(\xi)}{z-\xi} d \xi=I I I_{1}+I I I_{2} .
\end{aligned}
$$

On the one hand,

$$
\left|I I I_{2}\right| \leq \frac{1}{\pi} \int_{\Gamma \backslash \Gamma_{z, 2 \epsilon}}|T f(\xi)| \frac{\left|G_{z, \epsilon}(\xi)\right|}{|z-\xi|} d \mu(\xi) \lesssim \epsilon \int_{\Gamma \backslash \Gamma_{z, 2 \epsilon}} \frac{|T f(\xi)|}{|z-\xi|^{2}} d \mu(\xi) \lesssim M(T f)(z),
$$

where the last inequality is shown by splitting the integral over the sets

$$
\Gamma_{z, 2^{k+1} \epsilon} \backslash \Gamma_{z, 2^{k} \epsilon}, \quad k=1,2,3, \ldots
$$


On the other hand,

$$
\left|I I I_{1}\right|=|B(z, \epsilon)| \frac{1}{\pi} \int_{\Gamma \backslash \Gamma_{z, 2 \epsilon}} \frac{|T f(\xi)|}{|\xi-z|} d \mu(\xi) \lesssim \omega(2 \epsilon) \int_{\Gamma \backslash \Gamma_{z, 2 \epsilon}} \frac{|T f(\xi)|}{|z-\xi|} d \mu(\xi) .
$$

To estimate the last integral, we also split it over the sets

$$
\Gamma_{z, 2^{k+1} \epsilon} \backslash \Gamma_{z, 2^{k} \epsilon}, \quad k=1,2,3, \ldots
$$

Notice that, for $k$ big enough, $\Gamma_{z, 2^{k} \epsilon}=\Gamma$, and so $\Gamma_{z, 2^{k+1} \epsilon} \backslash \Gamma_{z, 2^{k} \epsilon}=\emptyset$. Precisely, this holds for all $k$ such that $2^{k} \epsilon>2 L$, which is equivalent to

$$
k>\frac{\log \frac{2 L}{\epsilon}}{\log 2} .
$$

As a result, if we denote by $k_{0}(\epsilon)$ the smallest integer $k$ that satisfies the previous inequality, we have

$$
\begin{aligned}
\int_{\Gamma \backslash \Gamma_{z, 2 \epsilon}} \frac{|T f(\xi)|}{|z-\xi|} d \mu(\xi) & =\sum_{k=1}^{k_{0}(\epsilon)} \int_{\Gamma_{z, 2^{k+1}} \backslash \Gamma_{z, 2^{k} \epsilon}} \frac{|T f(\xi)|}{|z-\xi|} d \mu(\xi) \\
& \lesssim \sum_{k=1}^{k_{0}(\epsilon)} \frac{1}{2^{k} \epsilon} \int_{\Gamma_{z, 2^{k+1} \epsilon} \backslash \Gamma_{z, 2^{k_{\epsilon}}}}|T f(\xi)| d \mu(\xi) \\
& \leq 4 \sum_{k=1}^{k_{0}(\epsilon)} \frac{1}{2 \cdot 2^{k} \epsilon} \int_{\Gamma_{z, 2^{k+1}}}|T f(\xi)| d \mu(\xi) \leq 4 k_{0}(\epsilon) M(T f)(z) .
\end{aligned}
$$

As a result,

$$
\left|I I I_{1}\right| \lesssim \omega(2 \epsilon) k_{0}(\epsilon) M(T f)(z) \lesssim \omega(2 \epsilon)\left|\log \frac{2 L}{\epsilon}\right| M(T f)(z)
$$

Gathering the estimates for $|I|,|I I|,\left|I I I_{1}\right|$ and $\left|I I I_{2}\right|$, we have

$$
\left|T_{\epsilon} f(z)\right| \lesssim M^{2}(T f)(z)+\omega(2 \epsilon)\left|\log \frac{2 L}{\epsilon}\right| M(T f)(z)
$$

From this, it follows that, if $\omega$ is such that $\omega(2 \epsilon)|\log \epsilon|$ stays bounded as $\epsilon \rightarrow 0$, then we have

$$
\left|T_{\epsilon} f(z)\right| \lesssim M^{2}(T f)(z)
$$

Thus, we have proved the following result:

Theorem 5. With the notation established in this section, suppose $\gamma^{\prime}$ has a modulus of continuity $\omega$ such that $\omega(\epsilon)|\log \epsilon|$ stays bounded as $\epsilon \rightarrow 0$ (this happens, for example, if $\gamma \in \mathcal{C}^{1+\delta}$ for some $\left.\delta>0\right)$. Then, there exists a constant $c>0$ such that, for all $f \in L^{2}(\Gamma, d \mu)$ and all $z \in \Gamma$,

$$
T_{*} f(z) \leq c M^{2}(T f)(z) .
$$

We want to remark, finally, that a totally analogous result holds if one considers the truncated operators given by

$$
\tilde{T}_{\epsilon} f(z)=\frac{1}{\pi i} \int_{\Gamma \backslash B(z, \epsilon)} \frac{f(\xi)}{\xi-z} d \xi
$$




\section{References}

[1] Calderón, A. P.: Cauchy integrals on Lipschitz curves and related operators. - Proc. Nat. Acad. Sci. USA 74, 1977, 1324-1327.

[2] Coifman, R. R., A. McIntosh, and Y. Meyer: L'intégrale de Cauchy définit un opérateur borné sur $L^{2}$ pour les courbes lipschitziennes. - Ann. of Math. (2) 116, 1982, 361-387.

[3] David, G., and S. Semmes: Singular integrals and rectifiable sets in $\mathbf{R}^{n}$ : Audelàs des graphes lipschitziens. - Astérisque 193, Société Mathématique de France, 1991.

[4] Duonndikoetxea, J.: Fourier analysis. - Grad. Stud. Math. 29, Amer. Math. Soc., 2001.

[5] Escauriaza, L.: - Manuscript, 2012.

[6] Grafakos, L.: Modern Fourier analysis. - Grad. Texts in Math. 250, second edition, SpringerVerlag, 2008.

[7] Mateu, J., J. Orobitg, C. Pérez, and J. Verdera: New estimates for the maximal singular integral. - Int. Math. Res. Not. IMRN 19, 2010, 3658-3722.

[8] Mateu, J., J. Orobitg, and J. Verdera: Estimates for the maximal singular integral in terms of the singular integral: the case of even kernels. - Ann. of Math. (2) 174:3, 2011, 14291483.

[9] Mateu, J., and J. Verdera: $L^{p}$ and weak $L^{1}$ estimates for the maximal Riesz transform and the maximal Beurling transform. - Math. Res. Lett. (13) 5-6, 2006, 957-966.

[10] Tolsa, X.: Analytic capacity, the Cauchy transform, and non-homogeneous CalderónZygmund theory. - Preliminary version available at http://mat.uab.es/ xtolsa/llibreweb.pdf.

[11] Wilson, M.: Weighted Littlewood-Paley theory and exponential-square integrability. - Lecture Notes in Math. 1924, Springer-Verlag, 2008.

Received 26 July 2012 • Accepted 11 December 2012 